\title{
AN EXTENSION OF HARTOGS' THEOREM FOR DOMAINS WHOSE BOUNDARY IS NOT SMOOTH
}

\author{
DAVID G. SCHAEFFER ${ }^{1}$
}

\begin{abstract}
In this note we obtain an extension of Hartogs' theorem on analytic continuation inside a bounded domain in $C^{n}$ which requires no assumption on the smoothness of the boundary. The standard proof of Hartogs' theorem applies with the minor change of using a Whitney function as the boundary data.
\end{abstract}

Let $\Omega$ be a bounded domain in $C^{n}, n>1$, whose complement is connected. According to Hartogs' theorem, if $\partial \Omega$ is a surface of class $C^{2}$ and if $u \in C^{2}(\partial \Omega)$ satisfies the tangential Cauchy-Riemann equations on $\partial \Omega$, then $u$ is the restriction to $\partial \Omega$ of a function $U \in C^{1}(\bar{\Omega})$ analytic inside $\Omega$. In this note we observe that the smoothness condition $\partial \Omega \in C^{2}$ may be discarded in favor of the very mild hypothesis that $\Omega$ equals the interior of its closure, providing the boundary data are taken to be a Whitney function of class $C^{2}$ which satisfies all the Cauchy-Riemann equations on $\partial \Omega$. We remark that an ordinary function $u$ which satisfies the tangential Cauchy-Riemann equations on a smooth surface $S$ may be assigned a normal derivative to form a Whitney function on $S$ which satisfies all the Cauchy-Riemann equations.

We follow closely the proof of Hartogs' theorem in $\$ 2.3$ of Hörmander [1]. If $K$ is a compact set in $C^{n}$, let $\mathcal{E}^{m}(K)$ be the space of (complex-valued) Whitney functions on $K$ of class $C^{m}$. (Chapter I of Malgrange [2] contains a concise introduction to Whitney functions.) We use the notation $\nabla=\left(\partial / \partial z_{1}, \cdots, \partial / \partial \bar{z}_{1}, \cdots\right)$ for the gradient operator.

TheOREM. Let $\Omega$ be a bounded domain in $C^{n}, n>1$, such that $\Omega=\operatorname{Int}(\bar{\Omega})$ and $\Omega^{\prime}=C^{n} \sim \bar{\Omega}$ is connected. If $u \in \mathcal{E}^{2}(\partial \Omega)$ and if $\bar{\partial} u$ $=\nabla(\bar{\partial} u)=0$, then $u$ may be extended to a function $U \in \mathcal{E}^{1}(\bar{\Omega})$ analytic in $\Omega$.

Proof. By the Whitney extension theorem, there is a function $v \in C^{2}\left(\boldsymbol{C}^{n}\right)$ which is an extension of $u$. Define a differential form of type $(0,1)$,

Received by the editors July 18, 1969.

AMS Subject Classifications. Primary 3222.

Key Words and Phrases. Several complex variables, pseudo-convexity, Hartogs' Theorem, Whitney functions.

1 This research was partially supported by the National Science Foundation under NSF Grant GP-6761. 


$$
f=\bar{\partial} v \text { in } \Omega, \quad f=0 \text { on } C^{n} \sim \Omega .
$$

Then $f \in C^{1}\left(C^{n}\right), \bar{\partial} f=0$, and $f$ has compact support. By Theorem 2.3.1 of Hörmander, there is a function $w \in C_{c}^{1}\left(C^{n}\right)$ such that $\bar{\partial} w=f$. We note that $\partial \Omega^{\prime}=\partial \Omega$, because $\Omega=\operatorname{Int}(\bar{\Omega})$; it follows by unique continuation that $w \mid \partial \Omega=0$. Of course the function $U=v-w$ provides the desired analytic continuation of $u$.

It is a pleasure to acknowledge a stimulating conversation with Stephen Greenfield which led me to this problem, and I would like to thank Hugo Rossi for reading the paper before publication.

\section{REFERENCES}

1. L. Hörmander, An introduction to complex analysis in several variables, Van Nostrand, Princeton, N. J., 1966. MR 34 \#2933.

2. B. Malgrange, Ideals of differentiable functions, Tata Institute of Fundamental Research, Bombay and Oxford Univ. Press, London, 1967. MR 35 \#3446.

BRANDEIS UNIVERSITY 